\title{
Controlling the photonic band structure of optically driven cold atoms
}

\author{
Jin-Hui Wu, ${ }^{1, *}$ M. Artoni, ${ }^{2,3}$ and G. C. La Rocca ${ }^{4}$ \\ ${ }^{1}$ College of Physics, Jilin University, Changchun 130023, China \\ ${ }^{2}$ Department of Physics and Chemistry of Materials, Brescia University, Brescia, Italy \\ ${ }^{3}$ European Laboratory for Nonlinear Spectroscopy, Firenze, Italy \\ ${ }^{4}$ Scuola Normale Superiore and CNISM, Pisa, Italy \\ *Corresponding author: wujinhui0431@sina.com
}

Received May 13, 2008; revised September 1, 2008; accepted September 9, 2008; posted September 11, 2008 (Doc. ID 95937); published October 15, 2008

\begin{abstract}
An analytical method based on a two-mode approximation is here developed to study the optical response of a periodically modulated medium of ultracold atoms driven into a regime of standing-wave electromagnetically induced transparency. A systematic comparison with the usual approach based on the coupled MaxwellLiouville equations shows that our method is very accurate in the frequency region of interest. Our method, in particular, explains in a straightforward manner the formation of a well-developed photonic bandgap in the optical Bloch wave vector dispersion. For ultracold ${ }^{87} \mathrm{Rb}$ atoms nearly perfect reflectivity may be attained and a light pulse whose frequency components are contained within the gap is seen to be reflected with little loss and deformation. (c) 2008 Optical Society of America
\end{abstract}

OCIS codes: $270.1670,160.5298,190.4223$.

\section{INTRODUCTION}

Laser induced quantum coherence schemes lie at the heart of quantum physics, especially for their applications to light propagation control, enhancement of resonant optical nonlinearity, and state transfer between light and atoms, just to mention a few. The main impetus for such an intense activity comes, however, from potential applications to optical memories, optical computing, and quantum information storage and processing. One of these schemes relies on electromagnetically induced transparency (EIT) [1,2], which refers to the absorption suppression of a probe laser due to the quantum coherence established through a strong monochromatic coupling laser. With a static cw coupling field, one can easily achieve ultraslow light accompanied by little absorption in atomic gases or solid materials [3,4]. By a dynamic modulation of the cw coupling field in time one can instead transfer the quantum state of a probe pulse to the dressed media and retrieve it after storage [5-7]. Storage times in this case are of the order of milliseconds and typically limited by spin coherence dephasing processes. By slightly disturbing the conditions for EIT through an auxiliary level greatly enhanced nonlinear interaction [8-10] may also be achieved, which has been exploited to devise efficient quantum phase gates [11,12] and all-optical switching [13].

An important step toward light storage was taken by employing coupling fields in a standing-wave (SW) configuration to periodically modulate the dispersive [14] properties of a medium and induce a photonic bandgap in the medium. The absorptive properties may also be periodically modulated and this has been exploited to trap [15] a probe pulse in hot atomic vapors of ${ }^{87} \mathrm{Rb}$. Such stationary light pulses may be used, e.g., to devise interest- ing schemes to enhance the Kerr-like interaction between a probe and another signal pulse stored in the spin coherence [16] or to achieve quantum transport of single photons or quantum states [17]. Light storage in dynamically controlled optical lattices have been thoroughly studied and detailed theoretical treatments have been given in terms of coupled Maxwell-Liouville equations [18-21] or using the dark-state polariton picture associated with EIT [5].

The generation of stationary light pulses in all-optical controlled bandgap media makes it clearly unnecessary to design photonic crystals [22] with predetermined bandgap structures, and thus may be very useful for controlling the light flow and interactions. Unfortunately, there exists remarkable diffusion and loss due to the imperfect reflectivity and residual absorption [18]. One possible way to reduce diffusion and loss consists in improving the reflectivity of the dynamically induced photonic bandgap $[23,24]$. Recent work based on the transfer-matrix method shows that well-developed photonic bandgaps may open up in ultracold atomic gases and impurity doped solids [25-27] due to the periodically modulated refractive index accompanied by negligible absorption when a specific EIT configuration is adopted. Other optical methods, e.g., through the ac Stark effect and the coherently enhanced refractive index, have also been proposed to attain controllable photonic bandgaps in semiconductor quantum wells [28,29].

In this paper, we investigate the optical response of ultracold ${ }^{87} \mathrm{Rb}$ atoms driven by a stationary SW coupling field both to a static ( $c w)$ and to a time-dependent (pulse) probe field. Starting from the atomic steady-state susceptibility experienced by the probe, we first derive in Section 2 the Bloch eigenstates of the electromagnetic field 
within a two-mode approximation, from which analytical expressions for the bandgap structure and reflectivity and transmissivity can be obtained. For appropriate parameters we observe rather well-developed bandgaps and nearly perfect reflection of an incident cw probe, in agreement with the results obtained using the same dressed susceptibility within a transfer-matrix approach not relying on the two-mode approximation. The response to a light pulse is also examined by resorting to a set of coupled Maxwell-Liouville equations, which is a powerful tool [18-21] for describing the nonlinear interaction of light fields in such a dressed medium and are here used to test the validity of the two-mode approximation in the appropriate limit. This is done in Section 3 where we study the time evolution of the reflection and transmission processes of a long square probe pulse. In the long time limit we recover the results for the reflectivity and transmissivity spectra found in Section 2. Numerical calculations show that the steady-state reflectivity and transmissivity can only be observed after a sufficiently long oscillating transient time. We also find that a probe pulse of narrow bandwidth can be either perfectly reflected with a shorter time delay or partially transmitted with a longer time delay depending on whether its central frequency falls in the middle of the bandgap or not. We finally investigate significant effects of penetration even in a regime of perfect reflection in which case the time-dependent intensity distribution of the probe pulse may be quite complicated due to the interference among the forward (FD) and backward (BD) probe components.

\section{CASE OF A CONTINUOUS WAVE PROBE FIELD}

We here consider an ensemble of $\Lambda$-type three-level atoms with an excited state level $|3\rangle$ and two lower levels $|1\rangle$ and $|2\rangle$. As shown in Fig. 1 a probe of frequency $\omega_{p}$ and wave vector $k_{p}$ and a coupling field of frequency $\omega_{c}$ and wave vector $k_{c}$ couple the two lower states to the common excited state. In the interaction picture, the atomic dynamical evolution can be described by the following Hamiltonian:

$$
H=\hbar \omega_{31}|3\rangle\left\langle 3\left|+\hbar \omega_{21}\right| 2\right\rangle\langle 2|+H_{\text {int }},
$$

where

$$
H_{\text {int }}=-\hbar \Omega_{p} e^{-i \Delta_{p} t}|3\rangle\left\langle 1\left|-\hbar \Omega_{c} e^{-i \Delta_{c} t}\right| 3\right\rangle\langle 2|+\text { H.c. }
$$

describes the interaction of the atoms with the probe and coupling fields. Here $\Delta_{p}=\omega_{p}-\omega_{31}$ and $\Delta_{c}=\omega_{c}-\omega_{32}$ denote detunings of the probe and coupling fields from the corresponding transition frequencies, whereas $\Omega_{p}=E_{p} d_{31} / 2 \hbar$ and $\Omega_{c}=E_{c} d_{32} / 2 \hbar$ denote the two fields's Rabi frequencies. Also, $E_{p}$ and $d_{31}$ are the probe electric field amplitude and the probe electric dipole moment with a similar notation for $E_{c}$ and $d_{32}$. If $\Gamma_{31}, \Gamma_{32}$, and $\Gamma_{21}$ denote the population decay rates and $\gamma_{12}=\Gamma_{21} / 2, \quad \gamma_{13}=\left(\Gamma_{31}+\Gamma_{32}\right) / 2$, and $\gamma_{23}$ $=\left(\Gamma_{31}+\Gamma_{32}+\Gamma_{21}\right) / 2$ the coherence decay rates, solutions of the Liouville's equation,

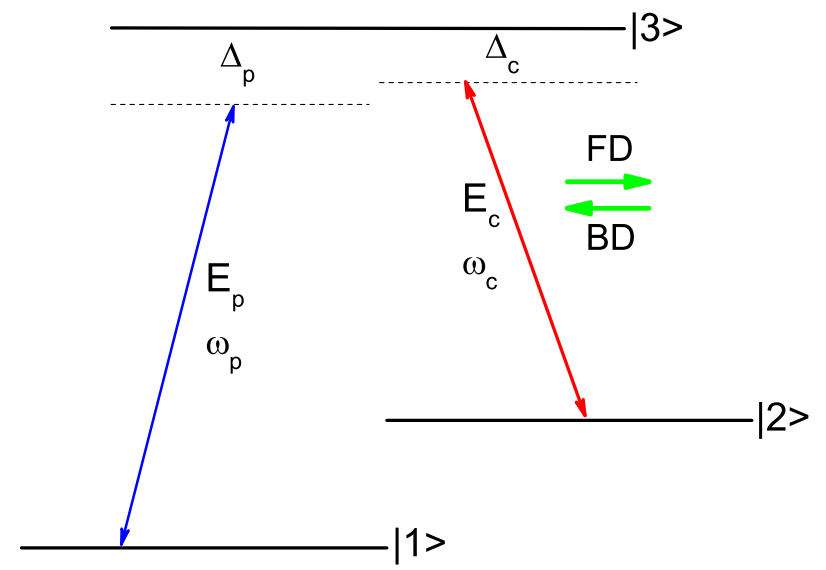

Fig. 1. (Color online) Schematic of a probe $\omega_{p}$ impinging on a three-level atom driven by an external coupling beam $\omega_{c}$, which is here retroreflected upon impinging on a mirror to form a SW pattern within the atomic sample. By slightly reducing the mirror reflectivity the FD and $\mathrm{BD}$ components of the SW intensity no longer vanishes at the nodes that are replaced by quasi-nodes. Furthermore, a misalignment $\theta$ between the FD and BD beam components, measured with respect to the direction $z$ where the two coupling beams are perfectly aligned, modifies the lattice periodicity as $\lambda_{c} / 2 \rightarrow \lambda_{c} / 2 \cos (\theta / 2)$.

$$
\begin{aligned}
& \frac{\partial \rho_{11}}{\partial t}=\Gamma_{31} \rho_{33}+\Gamma_{21} \rho_{22}+i \Omega_{p}^{*} \rho_{31}-i \Omega_{p} \rho_{13}, \\
& \frac{\partial \rho_{22}}{\partial t}=\Gamma_{32} \rho_{33}-\Gamma_{21} \rho_{22}+i \Omega_{c}^{*} \rho_{32}-i \Omega_{c} \rho_{23}, \\
& \frac{\partial \rho_{12}}{\partial t}=-\left[\gamma_{12}+i\left(\Delta_{p}-\Delta_{c}\right)\right] \rho_{12}+i \Omega_{p}^{*} \rho_{32}-i \Omega_{c} \rho_{13}, \\
& \frac{\partial \rho_{13}}{\partial t}=-\left[\gamma_{13}+i \Delta_{p}\right] \rho_{13}-i \Omega_{c}^{*} \rho_{12}+i \Omega_{p}^{*}\left(\rho_{33}-\rho_{11}\right), \\
& \frac{\partial \rho_{23}}{\partial t}=-\left[\gamma_{23}+i \Delta_{c}\right] \rho_{23}-i \Omega_{p}^{*} \rho_{21}+i \Omega_{c}^{*}\left(\rho_{33}-\rho_{22}\right),
\end{aligned}
$$

yield the macroscopic polarization $P=N d_{13} \rho_{31}$ and, in the limit of a weak probe, the susceptibility $\chi$ according to the relation $P=\varepsilon_{0} \chi_{p} E_{p}=N d_{13} \rho_{31}$ with $N$ being the atomic density. For a resonant coupling $\left(\Delta_{c} \equiv 0\right)$, the steady-state probe susceptibility obtained from Eqs. (3) can be written as

$$
\chi_{p}=\frac{N\left|d_{13}\right|^{2}}{2 \hbar \varepsilon_{0}} \frac{\Delta_{p}+i \gamma_{12}}{\left(\gamma_{12}-i \Delta_{p}\right)\left(\gamma_{13}-i \Delta_{p}\right)+\left|\Omega_{c}\right|^{2}} .
$$

This is a dressed susceptibility yielding a steep dispersion with a concomitant small absorption around $\Delta_{p}=0$, a characteristic feature of the EIT regime [1,2].

However, when the coupling field is in the form of a SW along the $z$ direction [30], the coupling Rabi frequency $\Omega_{c}$ in Eq. (4) becomes space-dependent and should be replaced by $\Omega_{c}(z)=\Omega_{c+} e^{i k_{c} z}+\Omega_{c-} e^{-i k_{c} z}$ with $\Omega_{c+}$ and $\Omega_{c-}$, taken to be real, representing the Rabi frequency amplitudes of the constituent FD and $\mathrm{BD}$ plane-wave compo- 
nents. The probe susceptibility, which depends now on $z$ through $\left|\Omega_{c}\right|^{2}(z)$, becomes a periodically modulated function of the form

$$
\chi_{p}(z)=\frac{N\left|d_{13}\right|^{2}}{2 \hbar \varepsilon_{0}} \frac{\mathcal{A}}{1+\mathcal{B} \cos \left(2 k_{c} z\right)},
$$

with

$$
\begin{aligned}
& \mathcal{A}=\frac{\Delta_{p}+i \gamma_{12}}{\Omega_{c+}^{2}+\Omega_{c-}^{2}-\Delta_{p}^{2}-i\left(\gamma_{12}+\gamma_{13}\right) \Delta_{p}+\gamma_{12} \gamma_{13}}, \\
& \mathcal{B}=\frac{2 \Omega_{c+} \Omega_{c-}}{\Omega_{c+}^{2}+\Omega_{c-}^{2}-\Delta_{p}^{2}-i\left(\gamma_{12}+\gamma_{13}\right) \Delta_{p}+\gamma_{12} \gamma_{13}} .
\end{aligned}
$$

This is an even function of $z$ with period $a=\pi / k_{c}=\lambda_{c} / 2$, where $\lambda_{c}$ is the coupling field's wavelength in the medium [31] and can be expanded as a cosine Fourier series [32], i.e.,

$$
\chi_{p}(z)=\chi_{0}+2 \sum_{n=1}^{\infty} \chi_{n} \cos \left(2 n k_{c} z\right)
$$

with

$$
\begin{aligned}
\chi_{n} & =\frac{k_{c}}{\pi} \frac{N \mathcal{A}\left|d_{13}\right|^{2}}{2 \hbar \epsilon_{o}} \int_{0}^{\pi / k_{c}} \frac{\cos \left(2 n k_{c} z\right) \mathrm{d} z}{1+\mathcal{B} \cos \left(2 k_{c} z\right)} \\
& =\frac{N \mathcal{A}\left|d_{13}\right|^{2}}{2 \hbar \epsilon_{0}} \frac{1}{\sqrt{1-\mathcal{B}^{2}}}\left(\frac{\sqrt{1-\mathcal{B}^{2}}-1}{\mathcal{B}}\right)^{n} .
\end{aligned}
$$

In a material that exhibits a periodically modulated susceptibility as in Eq. (5), on the other hand, the eigenfunctions of the electromagnetic field in the medium can be cast $[33,34]$ in the form

$$
E(z) \equiv \epsilon(z) e^{i \kappa z}=\left[\sum_{n=-\infty}^{\infty} \epsilon_{n} e^{i 2 n k_{c} z}\right] e^{i \kappa z},
$$

where $\epsilon(z)$ represents the complex amplitude of the probe electric field polarized along $x$ and propagating along $z$ with the Bloch wave-vector $\kappa$. Because $\epsilon(z)$ has the same periodicity of $\chi_{p}(z)$, it has been rewritten on the righthand side of Eq. (9) as a complex Fourier series of period $\pi / k_{c}$.

Eigenstates of the electromagnetic field satisfy Maxwell's wave equation so that for a monochromatic probe of frequency $\omega_{p}=c k_{p}$ in a medium with the susceptibility $\chi_{p}(z)$ one has

$$
\frac{\partial^{2} E(z)}{\partial z^{2}}+k_{p}^{2}\left[1+\chi_{p}(z)\right] E(z)=0 .
$$

Note that $k_{p}$ is here the vacuum probe wave vector at variance with $\kappa$ in Eq. (9) denoting instead the probe Bloch wave vector by which the steady-state optical response can be assessed in terms of the photonic band structure $[22,25]$. For the wave-vector $\kappa$ around $k_{c}$, the electric field in Eq. (9) is essentially determined by the two amplitudes $\epsilon_{0}$ and $\epsilon_{-1}$, which characterize the main Bragg components of the field in the periodically modulated medium. When Eq. (7) is inserted into Eq. (10) along with this two-component electric field (two-mode approximation), Eq. (10) reduces to a system of two coupled equations involving only the two susceptibility components $\chi_{0}$ and $\chi_{1}$. In matrix form this reads as

$$
\left(\begin{array}{cc}
k_{p}^{2}\left(1+\chi_{0}\right)-\kappa^{2} & k_{p}^{2} \chi_{1} \\
k_{p}^{2} \chi_{1} & k_{p}^{2}\left(1+\chi_{0}\right)-\left(\kappa-2 k_{c}\right)^{2}
\end{array}\right)\left(\begin{array}{c}
\epsilon_{0} \\
\epsilon_{-1}
\end{array}\right)=0 .
$$

It is convenient to set $\kappa=k_{c}+\mathrm{q}$, where q explores a small region around the Brillouin zone boundary $k_{c}$. To the lowest order in q one has

$$
\mathrm{q}_{ \pm} \simeq \pm \frac{1}{2 k_{c}} \sqrt{\left[k_{p}^{2}\left(1+\chi_{0}\right)-k_{c}^{2}\right]^{2}-k_{p}^{4} \chi_{1}^{2}} \equiv \pm q
$$

which is obtained from the determinant of Eq. (11) under the realistic assumption that $\left|\chi_{1}\right| \ll\left|1+\chi_{0}\right|$ and when half of the probe wavelength inside the medium matches the periodicity $a$. This yields two degenerate Bloch eigenstates of the electromagnetic field with electric field eigenfunctions $E^{ \pm}(z)$ in the form (two-mode approximation)

$$
\begin{aligned}
& E^{+}(z)=\epsilon_{0}^{+} e^{i \kappa_{+} z}+\epsilon_{-1}^{+} e^{-i \kappa_{-} z}, \\
& E^{-}(z)=\epsilon_{0}^{-} e^{i \kappa_{-} z}+\epsilon_{-1}^{-} e^{-i \kappa_{+} z},
\end{aligned}
$$

corresponding to the two Bloch wave vectors $\kappa_{ \pm}=k_{c} \pm q$ with eigenvectors $\epsilon_{\{0,-1\}}^{ \pm}$.

The electric field in Eq. (13) is seen to combine both FW and BW plane waves weighted by the corresponding eigenvectors. In fact, the off-diagonal term in Eq. (11) proportional to $\chi_{1}$ mixes into the Bloch eigenstates, respectively, the plane-wave $e^{i \kappa_{+} z}$ with $e^{-i \kappa_{-} z}$, and the plane-wave $e^{i \kappa_{-} z}$ with $e^{-i \kappa_{+} z}$. Furthermore, with the help of $\partial E_{p} / \partial z$ $=-(1 / c)\left(\partial B_{p} / \partial t\right)$, one has for the corresponding magnetic field eigenfunctions

$$
\begin{aligned}
& B^{+}(z)=\left(\epsilon_{0}^{+} \kappa_{+} e^{i \kappa_{+} z}-\epsilon_{-1}^{+} \kappa_{-} e^{-i \kappa_{-} z}\right) / k_{p}, \\
& B^{-}(z)=\left(\epsilon_{0}^{-} \kappa_{-} e^{i \kappa_{-} z}-\epsilon_{-1}^{-} \kappa_{+} e^{-i \kappa_{+} z}\right) / k_{p},
\end{aligned}
$$

while the total electromagnetic field may be written as

$$
\begin{aligned}
& E_{p}(z)=\alpha E^{+}(z)+\beta E^{-}(z), \\
& B_{p}(z)=\alpha B^{+}(z)+\beta B^{-}(z),
\end{aligned}
$$

with $\alpha$ and $\beta$ to be determined, and holding for a monochromatic probe in a periodically modulated medium.

For a sample of finite length $L$, typically containing a very large number of periods $a$, the reflection and transmission complex amplitudes $r$ and $t$ may be connected to the electric and magnetic fields at the inner $(z=0)$ and outer $(z=L)$ boundaries of the sample through the relations

$$
\begin{aligned}
& E_{p}(0)=(1+r) E_{\text {in }}(0), \\
& B_{p}(0)=(1-r) B_{\text {in }}(0), \\
& E_{p}(L)=t E_{\text {in }}(0),
\end{aligned}
$$




$$
B_{p}(L)=t B_{\text {in }}(0) \text {, }
$$

so that for an incident probe with amplitudes $E_{\text {in }}(0)$ $=B_{\text {in }}(0)$, taken here for simplicity to be unity, one obtains from Eq. (16)

$$
\begin{aligned}
& r=\alpha\left(\epsilon_{0}^{+}+\epsilon_{-1}^{+}\right)+\beta\left(\epsilon_{0}^{-}+\epsilon_{-1}^{-}\right)-1, \\
& r=1-\alpha \frac{\kappa_{+} \epsilon_{0}^{+}-\kappa_{-} \epsilon_{-1}^{+}}{k_{p}}-\beta \frac{\kappa_{-} \epsilon_{0}^{-}-\kappa_{+} \epsilon_{-1}^{-}}{k_{p}}, \\
& t=\alpha\left(\epsilon_{0}^{+}+\epsilon_{-1}^{+}\right) e^{i q L}+\beta\left(\epsilon_{0}^{-}+\epsilon_{-1}^{-}\right) e^{-i q L}, \\
& t=\alpha \frac{\kappa_{+} \epsilon_{0}^{+}-\kappa_{-} \epsilon_{-1}^{+}}{k_{p}} e^{i q L}+\beta \frac{\kappa_{-} \epsilon_{0}^{-}-\kappa_{+} \epsilon_{-1}^{-}}{k_{p}} e^{-i q L},
\end{aligned}
$$

where the appropriate expressions for the boundary values of the fields $E_{p}$ and $B_{p}$ as obtained from Eq. (15) have been used. The introduction of the ratios

$$
X_{ \pm} \equiv \frac{\epsilon_{0}^{ \pm}}{\epsilon_{-1}^{ \pm}}=\frac{-\chi_{1}}{\left(1+\chi_{0}\right)-\left(2 \kappa_{ \pm} k_{c}+k_{c}^{2}\right) / k_{p}^{2}},
$$

and the elimination from Eq. (17) of the two unknowns $\alpha$ and $\beta$ finally enables one to arrive at the two-mode approximation reflection and transmission coefficients

$$
\begin{aligned}
& r=\frac{2 A_{-}\left(1+X_{+}\right) e^{i q L}-2 B_{-}\left(1+X_{-}\right) e^{-i q L}}{A_{-} B_{+} e^{i q L}-A_{+} B_{-} e^{-i q L}}-1, \\
& t=\frac{2 A_{-}\left(1+X_{+}\right)-2 B_{-}\left(1+X_{-}\right)}{A_{-} B_{+} e^{i q L}-A_{+} B_{-} e^{-i q L}},
\end{aligned}
$$

where $A_{ \pm}=\left(1 \pm \kappa_{+} / k_{p}\right) X_{+}+\left(1 \mp \kappa_{-} / k_{p}\right) \quad$ and $\quad B_{ \pm}$ $=\left(1 \pm \kappa_{-} / k_{p}\right) X_{-}+\left(1 \mp \kappa_{+} / k_{p}\right)$.

We now proceed to examine characteristic photonic structures that may be induced in a sample of ultracold ${ }^{87} \mathrm{Rb}$ atoms. Levels $|1\rangle$ and $|2\rangle$ shown in Fig. 1 are here taken to be the hyperfine components $|F=1\rangle$ and $|F=2\rangle$ of the ground state $S_{1 / 2}$ while $|3\rangle$ is the hyperfine component $|F=1\rangle$ of the excited state $P_{3 / 2}$ with the relevant parameters given in the caption of Fig. 2 . We further assume all atoms are equally populated into the three magnetic sublevels of $\left|5 S_{1 / 2}, F=1\right\rangle$ and the probe and coupling fields are both linearly polarized with high purities. In this case, the three noninteracting lambda systems associated with specific magnetic quantum numbers act as a single one because no atoms are pumped into $\left|5 P_{3 / 2}, F=1\right\rangle$ and thus no population is transferred between these lambda systems. For this atomic structure, we start by examining the Bloch wave-vector modes $\kappa_{ \pm}$near the Brillouin zone band edge and show in Fig. $2 \operatorname{Im}\left(\kappa_{ \pm} a / \pi\right)$ and $\operatorname{Re}\left(\kappa_{ \pm} a / \pi\right.$ -1) as obtained from Eq. (12). A photonic stop-band of $\sim 0.5 \mathrm{MHz}$ is seen to open up when $\kappa \rightarrow k_{c}=\pi / a$, due to the off-diagonal term in Eq. (11) proportional to $\chi_{1}$ [35]. Color changing of the two curves in Fig. 2(a) means that the two modes $\kappa_{ \pm}$exchange the values of their imaginary parts, which is, however, immaterial.

We then study the reflectivity $R=|r|^{2}$ and the transmitivity $T=|t|^{2}$ profiles as obtained from Eqs. (19) when Eq. (12) is used. These are plotted for different sample widths

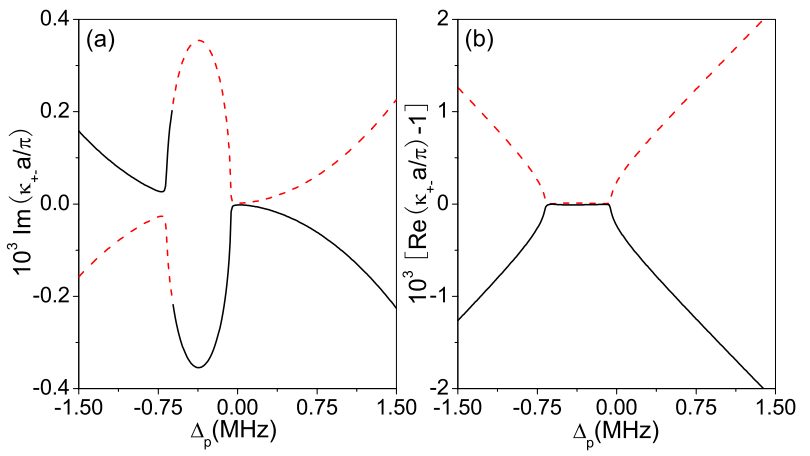

Fig. 2. (Color online) Photonic bandgap structures in a sample of ultracold ${ }^{87} \mathrm{Rb}$ atoms as obtained from Eq. (12). The black-solid and red-dashed curves correspond to $\kappa_{+}$and $\kappa_{-}$. The atomic parameters are $\Gamma_{31}=\Gamma_{32}=6.0 \mathrm{MHz}, \Gamma_{21}=2.0 \mathrm{kHz}$, and $N=10^{13} \mathrm{~cm}^{-3}$, while the FD and BD coupling beam components have Rabi frequencies $\Omega_{c+}=30.0 \mathrm{MHz}$ and $\Omega_{c-}=25.0 \mathrm{MHz}$ and are slightly misaligned $(\theta=45.0 \mathrm{mrad})$. The probe transition wavelength is $\lambda_{31}$ $=780.792 \mathrm{~nm}$ while for the coupling beam we have $\lambda_{32}$ $=780.778 \mathrm{~nm}$.

in Fig. 3 where over $95 \%$ bandgap reflectivity is attained for the wider atomic sample $(2.0 \mathrm{~mm})$ yet it gradually degrades as the sample width decreases. Comparison of Figs. 3(a) and 3(b) yields, in addition, very low values of the residual absorption $(A=1-R-T<5 \%)$ in the bandgap region. This ultimately hinges on the fact that EIT is pretty well-established within the whole sample owing to a coupling field that is sufficiently strong even at the SW quasinodes $\left(\Omega_{c \min }=5.0 \mathrm{MHz}\right)$. Moreover, the stop-band width where the reflectivity is homogeneously over $95 \%$ may be increased from $\sim 0.5$ to $\sim 3.5 \mathrm{MHz}$ if one uses a twice stronger coupling field misaligned at a larger angle (see Fig. 4).

The two-mode approximation described in this section and based on the use of the dressed susceptibility of Eq. (4) gives a very simple and transparent description of both photonic band structure and linear optical spectra in the case of a time-independent pump field. It is here appropriate to check the validity of the two-mode approximation by comparison with an exact treatment using the same dressed susceptibility as developed in [25,34]. Exploiting the system periodicity as in Eqs. (5) and (9) without any approximation, both the photonic bands of the in-
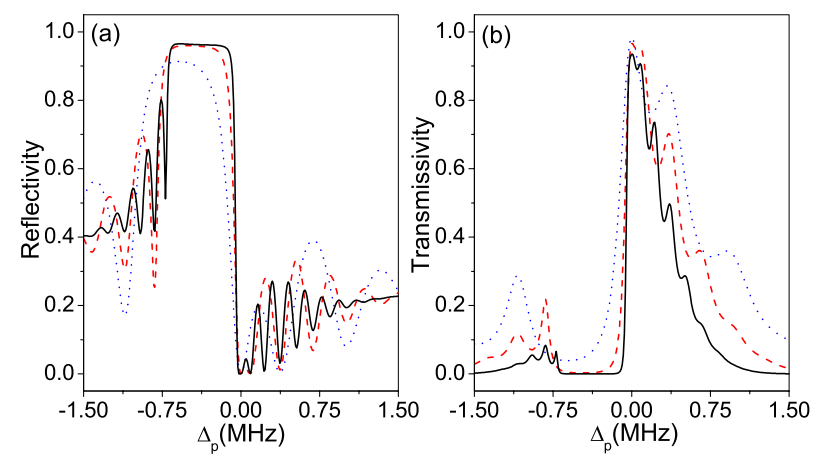

Fig. 3. (Color online) Probe reflectivity $(R)$ and transmissivity (T) as obtained from Eq. (19) within the two-mode approximation for an ultracold ${ }^{87} \mathrm{Rb}$ sample of width $L=2.0 \mathrm{~mm}$ (black-solid curve), $L=1.0 \mathrm{~mm}$ (red-dashed curve) and $L=0.5 \mathrm{~mm}$ (bluedotted curve). Other parameters are the same as in Fig. 2. 

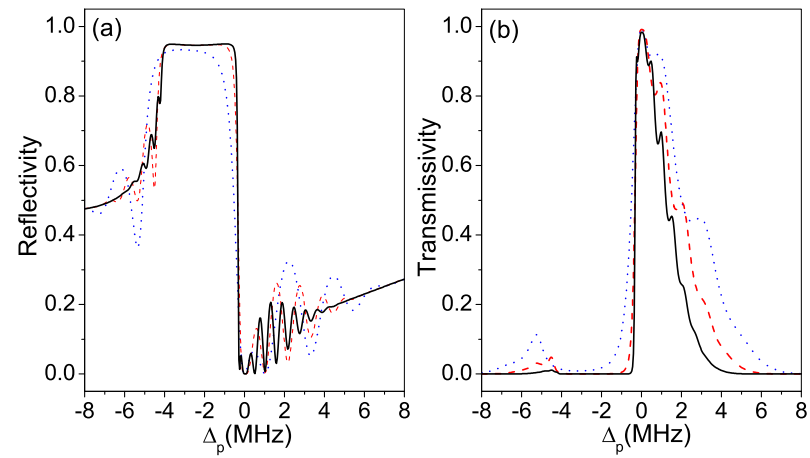

Fig. 4. (Color online) Probe reflectivity $(R)$ and transmissivity $(T)$ as obtained from Eq. (19) in an ultracold ${ }^{87} \mathrm{Rb}$ sample of width $L=2.0 \mathrm{~mm}$ (black-solid curve), $L=1.0 \mathrm{~mm}$ (red-dashed curve) and $L=0.5 \mathrm{~mm}$ (blue-dotted curve). $\Omega_{c+}=60.0 \mathrm{MHz}, \Omega_{c}^{-}$ $=50.0 \mathrm{MHz}$, and $\theta=55.0 \mathrm{mrad}$, while other parameters are the same as in Fig. 2.
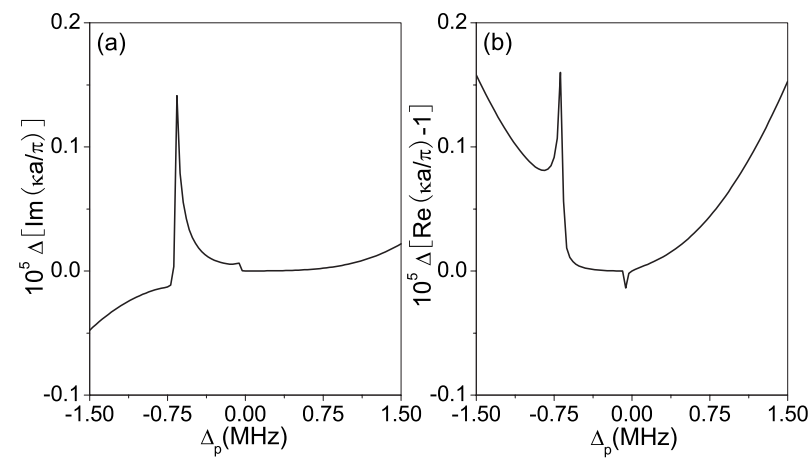

Fig. 5. Difference between the photonic energy bands obtained within the two-mode approximation (Fig. 2) and the exact ones obtained from the transfer-matrix approach $[25,36]$. All parameters are the same as in Fig. 2.

finite periodic medium and the reflectivity and transmittivity spectra of a finite sample can be obtained from the numerical calculation of the single period transfer matrix [36] for the probe electric field. The difference from such exact numerical calculations and the results presented in Figs. 2 and 3 are shown, respectively, in Figs. 5 and 6 . We can see a difference ranging between $0.1 \%$ and $1 \%$, which amounts to a $1 \%$ discrepancy at most between the approximate and exact results. Such a check

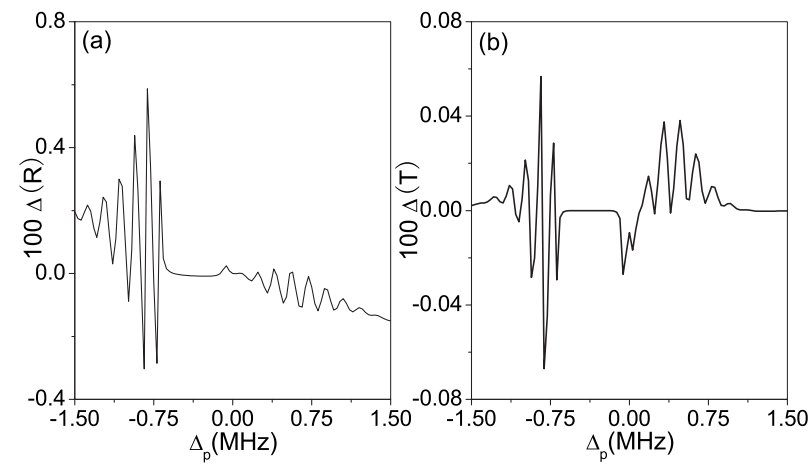

Fig. 6. Difference between the probe reflectivity $(R)$ and transmissivity $(T)$ obtained within the two-mode approximation (Fig. 3 ) and the exact ones obtained from the transfer-matrix approach for a $L=2.0 \mathrm{~mm}$ long sample $[25,36]$. Other parameters are the same as in Fig. 2. proves that the two-mode approximation yields, at least in the vicinity of the photonic bandgap, very accurate results. In Section 3 a similar two-mode approximation will be implemented to directly solve the coupled MaxwellLiouville equations.

\section{CASE OF A PULSED PROBE FIELD}

When dealing with a time-dependent coupling field, it is necessary to resort to the density matrix equations coupled with the Maxwell's wave equations (MaxwellLiouville equations). This fairly well-known approach [18-21] is here adopted to verify the validity of the twomode approximation discussed in Section 2. If on one hand indeed Maxwell-Liouville equations in the limit of a constant coupling field can be employed to evaluate the susceptibility components $\chi_{n}$ that are then to be compared with those given in Eq. (8), steady-state solutions of the Maxwell-Liouville equations may on the other hand be used to derive the reflectivity and transmissivity to be in turn compared with those found instead from Eq. (19).

In the limit of a weak probe one can set $\rho_{11}=1$ and $\rho_{22}$ $=\rho_{33}=\rho_{23} \simeq 0$ in Eq. (3) and just retain the equations for the two coherences $\rho_{12}$ and $\rho_{13}$, i.e.,

$$
\begin{aligned}
& \frac{\partial \rho_{12}}{\partial t}=-\left[\gamma_{12}+i\left(\Delta_{p}-\Delta_{c}\right)\right] \rho_{12}-i \Omega_{c} \rho_{13}, \\
& \frac{\partial \rho_{13}}{\partial t}=-\left[\gamma_{13}+i \Delta_{p}\right] \rho_{13}-i \Omega_{c}^{*} \rho_{12}-i \Omega_{p}^{*},
\end{aligned}
$$

with Rabi frequencies $\Omega_{c}=\Omega_{c+} e^{i k_{c} z}+\Omega_{c-} e^{-i k_{c} z}$ and $\Omega_{p}$ $=\Omega_{p+} e^{i k_{c} z}+\Omega_{p-} e^{-i k_{c} z}$ and associated probe and coupling electric fields

$$
\begin{aligned}
& E_{p}(z, t)=\left(E_{p+} e^{i k_{c} z}+E_{p-} e^{-i k_{c} z}\right) e^{-i \omega_{p} t}, \\
& E_{c}(z, t)=\left(E_{c+} e^{i k_{c} z}+E_{c-} e^{-i k_{c} z}\right) e^{-i \omega_{c} t} .
\end{aligned}
$$

Here $E_{p_{ \pm}}$and $E_{c \pm}$ are the space- and time-dependent slowly varying envelopes. To make comparisons with the results of Section 2 we here take $E_{c \pm} \rightarrow$ constant and $\Delta_{c}$ $\rightarrow 0$. It is to be stressed that the expression for the probe field in terms of the envelopes $E_{p \pm}(z, t)$ is not of the form given in Eq. (15) for a monochromatic probe.

By writing the spin $\left(\rho_{12}\right)$ and optical $\left(\rho_{13}\right)$ coherence, respectively, as

$$
\begin{aligned}
& \rho_{12}=\sum_{n=-\infty}^{\infty} \rho_{12}^{(n)} e^{i 2 n k_{c} z}, \\
& \rho_{13}=\sum_{n=-\infty}^{\infty} \rho_{13}^{(n)} e^{i(2 n+1) k_{c} z},
\end{aligned}
$$

one arrives at an infinite set of mutually coupled equations for their Fourier components, i.e.,

$$
\frac{\partial \rho_{12}^{(n)}}{\partial t}=-\gamma_{12}^{\prime} \rho_{12}^{(n)}-i \Omega_{c-} \rho_{13}^{(n)}-i \Omega_{c+} \rho_{13}^{(n-1)},
$$




$$
\begin{aligned}
\frac{\partial \rho_{13}^{(n)}}{\partial t}= & -\gamma_{13}^{\prime} \rho_{13}^{(n)}-i \Omega_{c-}^{*} \rho_{12}^{(n)}-i \Omega_{c+}^{*} \rho_{12}^{(n+1)}-i \Omega_{p-}^{*} \delta_{n, 0} \\
& -i \Omega_{p+}^{*} \delta_{n,-1}
\end{aligned}
$$

where $\gamma_{12}^{\prime}=\gamma_{12}+i \Delta_{p}$ and $\gamma_{13}^{\prime}=\gamma_{13}+i \Delta_{p}$. Sufficiently accurate solutions may be obtained upon truncating Eqs. (23) as, for instance, evaluating the first two components of the steady-state probe susceptibility through the equations

$$
\begin{aligned}
& \Omega_{p+} \chi_{0}+\Omega_{p-} \chi_{1}=\frac{N\left|d_{13}\right|^{2}}{2 \varepsilon_{0} \hbar} \rho_{31}^{(-1)}, \\
& \Omega_{p-} \chi_{0}+\Omega_{p+} \chi_{1}=\frac{N\left|d_{13}\right|^{2}}{2 \varepsilon_{0} \hbar} \rho_{31}^{(0)},
\end{aligned}
$$

which are as usual deduced from the relation [37] $P_{p}$ $=N d_{13} \rho_{31}$. Figures 7 and 8 show, in fact, that the approximate curves obtained from truncating Eqs. (23) at $|n|$ $=15$ deviate a little from the exact ones directly obtained from Eq. (8). Upon truncating Eqs. (23) at $|n|=30$ the curves obtained by using the two approaches completely overlap suggesting that higher Fourier components have in general little influence on the optical response of the periodic EIT medium. It can be seen that $\operatorname{Im}\left(\chi_{0}\right)\left[\operatorname{Re}\left(\chi_{0}\right)\right]$ and $\operatorname{Im}\left(\chi_{1}\right)\left[\operatorname{Re}\left(\chi_{1}\right)\right]$ approximately have the same absolute values yet with opposite signs around $\Delta_{p}=0$. This fact implies that the FW and BW plane-wave components in the probe field have the strongest mutual coupling, promoting the formation of a photonic bandgap.

As anticipated at the beginning of the section the reflectivity and transmissivity spectra may be inferred from the Maxwell-Liouville equations. When coupled to Eqs. (23), the Maxwell's wave equation

$$
\frac{\partial^{2} E_{p}}{\partial z^{2}}-\frac{1}{c^{2}} \frac{\partial^{2} E_{p}}{\partial t^{2}}=\mu_{0} \frac{\partial^{2} P}{\partial t^{2}},
$$
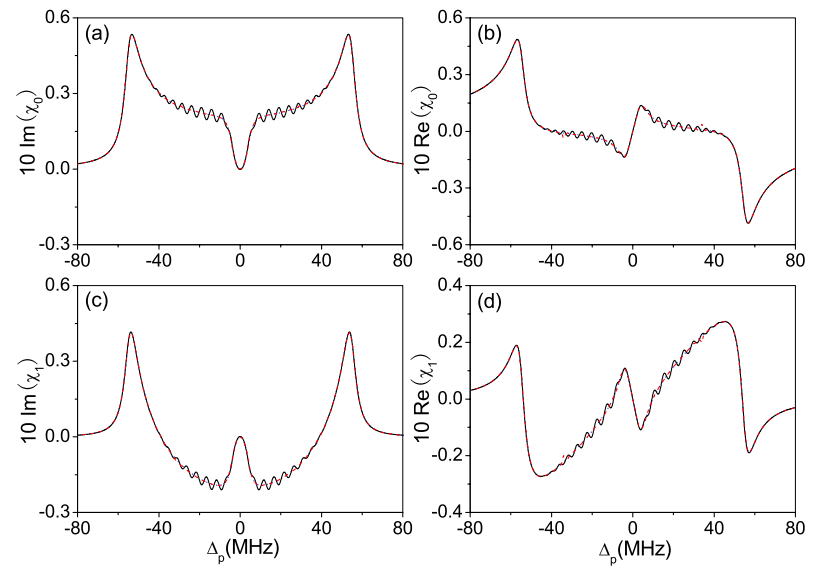

Fig. 7. (Color online) Zero- and first-order components of the probe susceptibility of a sample of ultracold ${ }^{87} \mathrm{Rb}$ atoms for the same parameters as in Fig. 2. The black-solid and red-dashed curves are, respectively, derived from Eqs. (23) truncated at $|n|$ $=15$ and 30, while the blue-dotted curves are from Eq. (8).

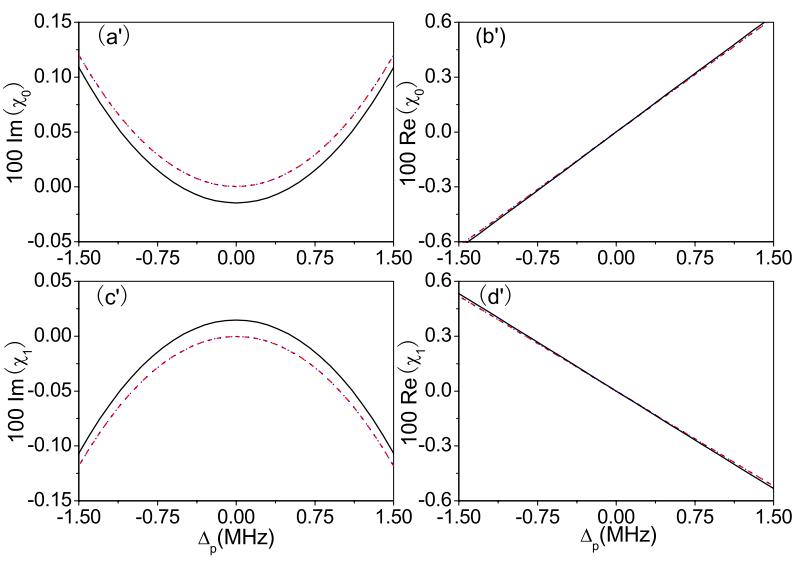

Fig. 8. (Color online) Central region blow-up of the probe susceptibility profiles of Fig. 7.

and its slowly varying envelope form

$$
\begin{gathered}
\left(\frac{\partial E_{p+}}{\partial z}+\frac{k_{p}}{c k_{c}} \frac{\partial E_{p+}}{\partial t}-\frac{i\left(k_{p}^{2}-k_{c}^{2}\right)}{2 k_{c}} E_{p+}\right) e^{+i k_{c} z}-\left(\frac{\partial E_{p-}}{\partial z}-\frac{k_{p}}{c k_{c}} \frac{\partial E_{p-}}{\partial t}\right. \\
\left.+\frac{i\left(k_{p}^{2}-k_{c}^{2}\right)}{2 k_{c}} E_{p-}\right) e^{-i k_{c} z}=i \frac{k_{p}^{2}}{k_{c}} \frac{N d_{13}}{2 \varepsilon_{0}} \rho_{31},
\end{gathered}
$$

become upon phase matching (mode coupling)

$$
\begin{aligned}
& \frac{\partial E_{p+}}{\partial z}+\frac{1}{c} \frac{\partial E_{p+}}{\partial t}=+i \Delta k E_{p+}+i \frac{N d_{13} k_{p}}{2 \varepsilon_{0}} \rho_{31}^{(0)}, \\
& \frac{\partial E_{p-}}{\partial z}-\frac{1}{c} \frac{\partial E_{p-}}{\partial t}=-i \Delta k E_{p-}-i \frac{N d_{13} k_{p}}{2 \varepsilon_{0}} \rho_{31}^{(-1)},
\end{aligned}
$$

when $k_{p} \approx k_{c} \approx\left(k_{p}+k_{c}\right) / 2$ and $\Delta k=k_{p}-k_{c}$. Boundary conditions on Eqs. (27) are chosen so that $E_{p+}(z=0, t)=E_{i t}(t)$ and $E_{p-}(z=L, t)=0$, with $E_{i t}(t)$ being the electric field amplitude of the incident probe pulse. Although only $\rho_{31}^{(0)}$ and $\rho_{31}^{(-1)}$ seem to contribute to the dynamics, one cannot neglect the other Fourier components of $\rho_{12}$ and $\rho_{13}$ in Eqs. (23) owing to their mutual coupling.

Maxwell-Liouville equations (27), along with Eq. (23), when truncated at $|n|=30$, enable one to assess with very high precision the propagation dynamics of a probe pulse incident upon the periodic EIT medium induced by a stationary SW coupling field. This is done in Fig. 9 where we calculate the time-dependent reflectivity and transmissivity at four typical probe detunings near the photonic bandgap for a long square probe pulse turned on at $t=0$ (see the insets). Both quantities experience a long-term oscillation and may exceed $100 \%$ before reaching the steady state. After sufficiently long times of $t$ $\sim 150-200 \mu \mathrm{s}$, the reflectivity and transmissivity reaches the steady state (see Fig. 10), showing very good agreement with those obtained from Eq. (19). This once again verifies the validity of the truncation at $|n|=30$ for Eqs. (23). It is here worth noting that likewise well-developed stop bands could be obtained at lower densities and shorter (longer) samples.

Then we begin to examine the reflected and transmitted parts of an incident Gaussian pulse as well as its FD and $\mathrm{BD}$ components in the periodic EIT medium. In 

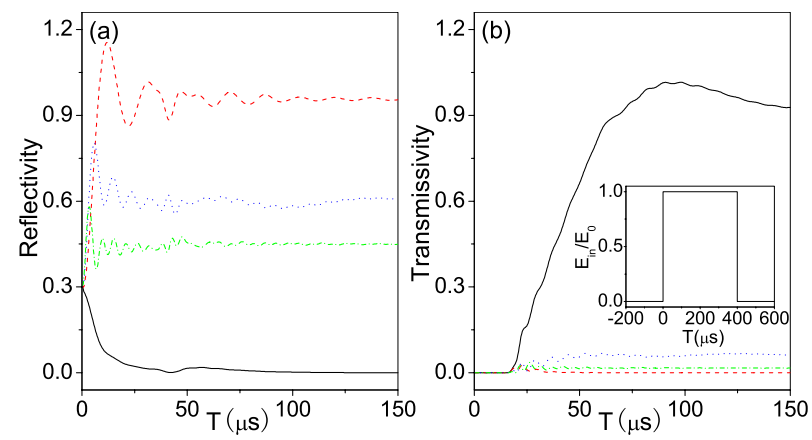

Fig. 9. (Color online) Reflected probe pulses seen at $z=0.0$ and transmitted probe pulses seen at $z=L$ for a long square pulse in a $L=2.0 \mathrm{~mm}$ long sample of ultracold ${ }^{87} \mathrm{Rb}$ atoms with $\Delta_{p}$ $=0.0 \mathrm{MHz}$ (black-solid curve), $-0.4 \mathrm{MHz}$ (red-dashed curve), $-0.8 \mathrm{MHz}$ (blue-dotted curve), and $-1.2 \mathrm{MHz}$ (green-dash-dotted curve), respectively. Other parameters are the same as in Fig. 2.

the following, we will assume that $E_{i t}(t)$ $=E_{0} \exp \left[-\left(t-T_{0}\right)^{2} /\left(4 \pi \delta T^{2}\right)\right]$ with the central probe detuning denoted by $\Delta_{p 0}$. Figure 11 shows that the reflected pulse at $z=0$ and the transmitted pulse at $z=L$ are quite sensitive to the central frequency $\Delta_{p 0}$ of the incident probe. When $\Delta_{p 0}$ is in the middle of the bandgap the reflected pulse has little attenuation and distortion compared with the incident probe except a short time delay $(\Delta t=2.0 \mu \mathrm{s})$. Conversely, it will experience more and more attenuation and distortion due to the increased transmission and/or absorption if $\Delta_{p 0}$ moves outward the bandgap.

In Fig. 12, we plot the field distributions at different times for the FD and BD probe components with $\Delta_{p 0}$ $=-0.4 \mathrm{MHz}$, and find that the FD and $\mathrm{BD}$ components penetrate deeply into the periodic EIT medium even if the reflectivity is nearly perfect. The typical scale of the field penetration is set by the imaginary part of the optical Bloch wave vector within the photonic bandgap. If the penetration length is defined as $L_{p}=1 /[2 \operatorname{Im}(\kappa)]$, one obtains $L_{p}=0.176 \mathrm{~mm}$ for $\Delta_{p}=-0.4 \mathrm{MHz}$ from Fig. 2(a), which is consistent with Fig. 12 where $I_{p+}(z$ $=0.185 \mathrm{~mm}) / I_{p+}(z=0.0 \mathrm{~mm})=1 / e$ at $T=25 \mu \mathrm{s}$. In Fig. 13 , with another central frequency $\Delta_{p 0}=0.0 \mathrm{MHz}$, we plot again the field distributions for the FD and $\mathrm{BD}$ probe components. We can see that, due to the imperfect reflec-
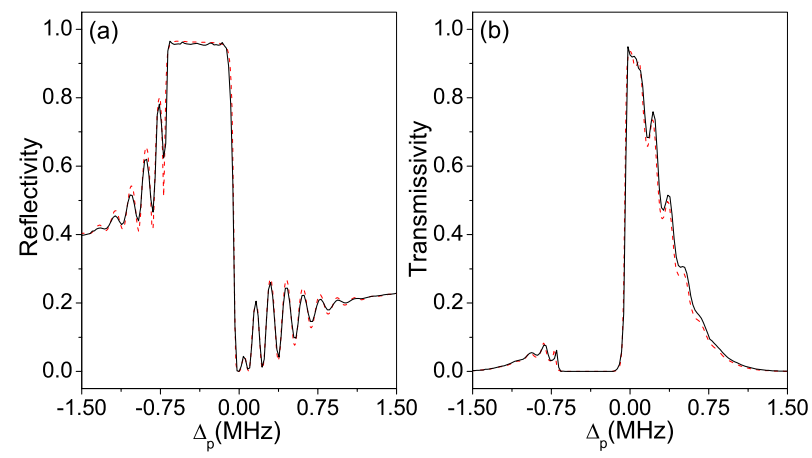

Fig. 10. (Color online) Steady-state reflectivity and transmissivity spectra in a $L=2.0 \mathrm{~mm}$ long sample of ultracold ${ }^{87} \mathrm{Rb}$ atoms. The black-solid curves are derived from the Maxwell-Liouville equations, i.e., from Eqs. (23) truncated at $|n|=30$ and Eqs. (27), while the red-dashed curves are from Eqs. (19) within the two-mode approximation. Other parameters are the same as in Fig. 2.

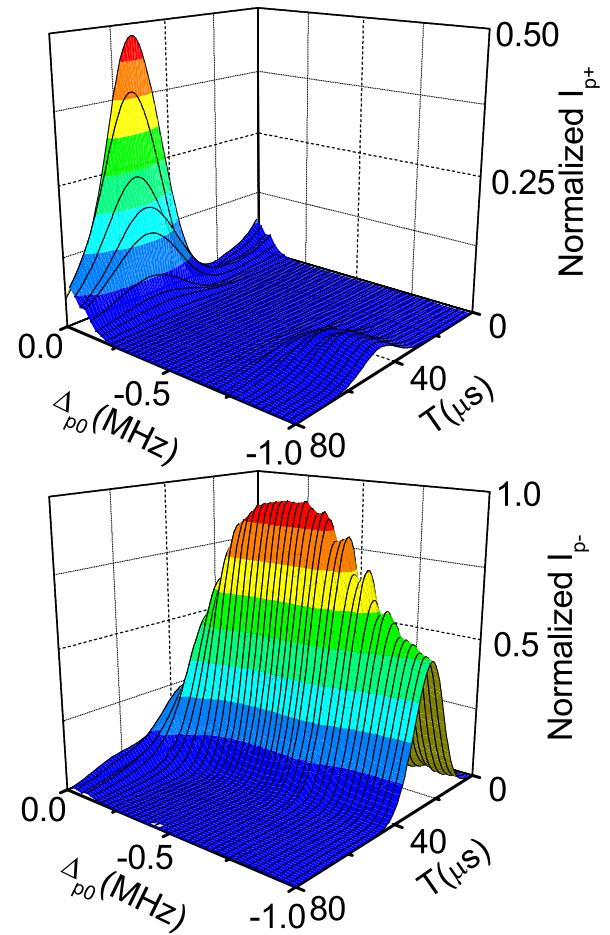

Fig. 11. (Color online) Transmitted components at $z=L$ (upper) and reflected components at $z=0$ (lower) of a weak probe pulse incident upon a $L=2.0 \mathrm{~mm}$ long sample of ultracold ${ }^{87} \mathrm{Rb}$ atoms with $T_{0}=25.0 \mu \mathrm{s}$ and $\delta T=4.0 \mu \mathrm{s}$. Other parameters are the same as in Fig. 2.

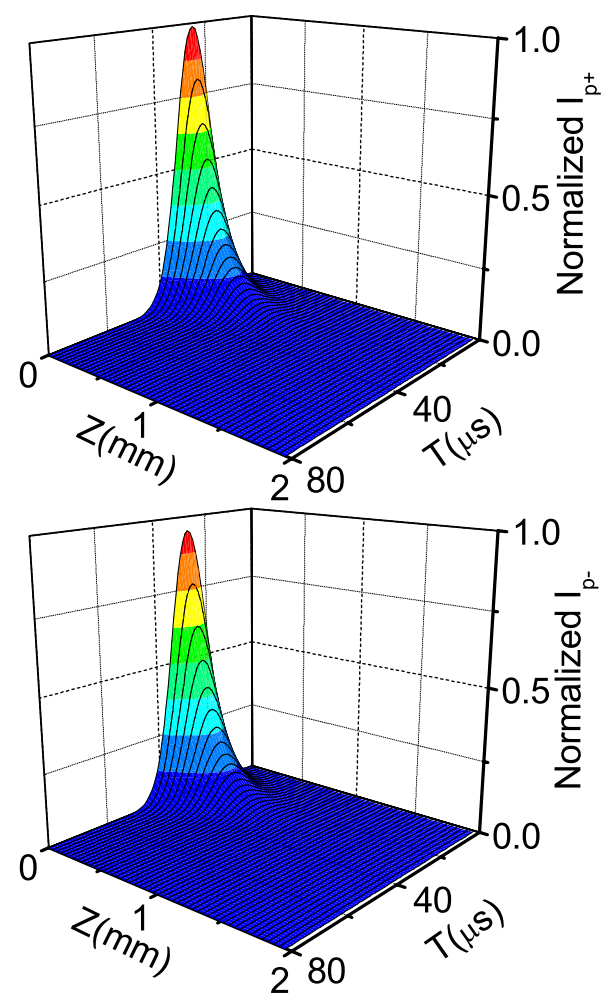

Fig. 12. (Color online) Scaled intensity distributions of the FD (upper) and BD (lower) probe pulses inside a sample of ultracold ${ }^{87} \mathrm{Rb}$ atoms with $\Delta_{p 0}=-0.4 \mathrm{MHz}$. Other parameters are the same as in Fig. 11. 


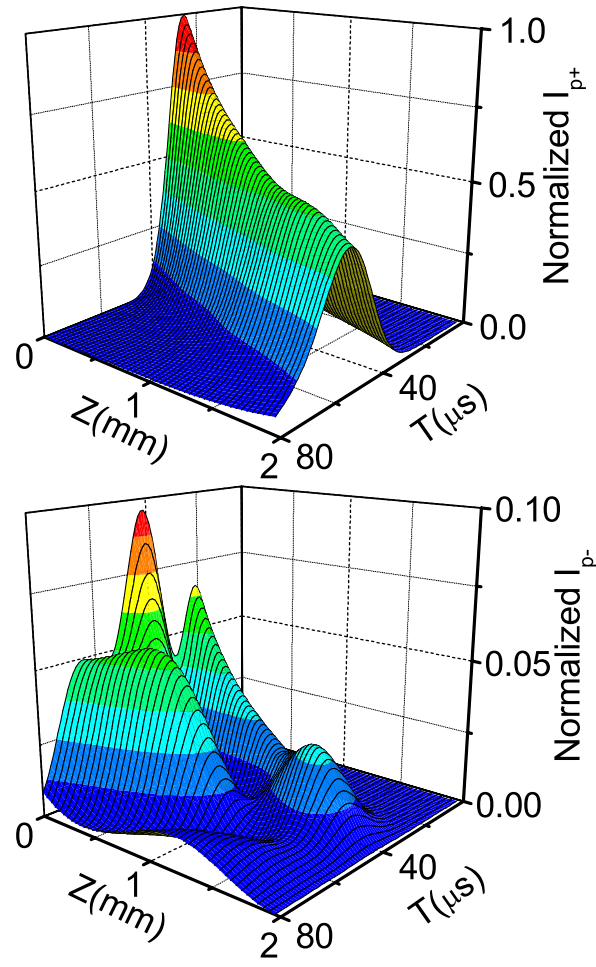

Fig. 13. (Color online) Scaled intensity distributions of the FD (upper) and $\mathrm{BD}$ (lower) probe pulses inside a sample of ultracold ${ }^{87} \mathrm{Rb}$ atoms with $\Delta_{p 0}=0.0 \mathrm{MHz}$. Other parameters are the same as in Fig. 11.

tivity, both FD and $\mathrm{BD}$ components can reach the end of the atomic sample, and their distributions change dramatically with time due to the interference resulting from the multiple feedback at the boundaries.

As long as the coupling field is time independent, the propagation dynamics of a probe pulse can also be described on the basis of the frequency dependent dressed susceptibility of Eq. (4). Using the Fourier-tansform technique, one first obtains the incident probe spectrum $E_{i f}\left(\Delta_{p}\right)=\int E_{i t}(t) e^{-i\left(\Delta_{p}-\Delta_{p 0}\right) t} \mathrm{~d} t$. Then, the reflected and transmitted spectra can be examined by $E_{r f}\left(\Delta_{p}\right)=E_{i f}\left(\Delta_{p}\right) \cdot r\left(\Delta_{p}\right)$ and $E_{t f}\left(\Delta_{p}\right)=E_{i f}\left(\Delta_{p}\right) \cdot t\left(\Delta_{p}\right)$ with $r\left(\Delta_{p}\right)$ and $t\left(\Delta_{p}\right)$ derived using the dressed susceptibility either within a two-mode approximation as described in Section 2 or from the exact numerical transfer-matrix method. Using the Fourier transform again, one finally has

$$
\begin{aligned}
& E_{r t}(t)=\int E_{r f}\left(\Delta_{p}\right) e^{i\left(t-T_{0}\right) \Delta_{p}} \mathrm{~d}\left(\Delta_{p}\right), \\
& E_{t t}(t)=\int E_{t f}\left(\Delta_{p}\right) e^{i\left(t-T_{0}\right) \Delta_{p}} \mathrm{~d}\left(\Delta_{p}\right),
\end{aligned}
$$

which just yields the amplitudes of the reflected and transmitted pulses. In Fig. 14, we show the incident and reflected pulses for two different central probe detunings inside or near the photonic bandgap. We can see that the Maxwell-Liouville equations and the dressed susceptibility approach once again generate the same results with very high precision. In a similar way, the two methods lead to the same results also for the examples shown in Figs. 9 and 11-13.
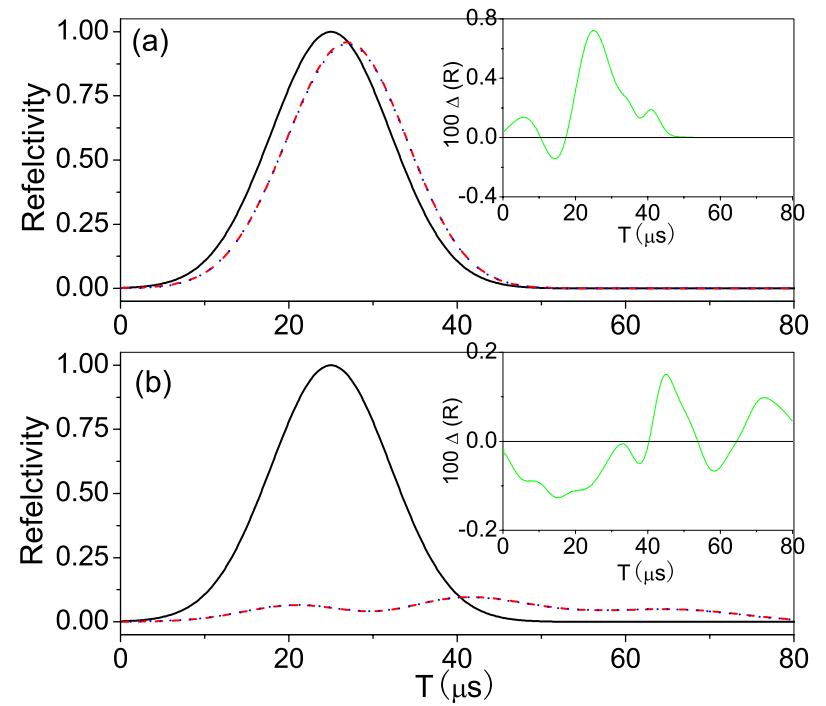

Fig. 14. (Color online) Reflected probe pulses seen at $z=0$ as obtained by using the dressed susceptibility (red-dashed curve) and the Maxwell-Liouville (blue-dotted curve) approach. The incident probe pulse (black-solid curve), which is here scaled to unity, has a carrier frequency $\Delta_{p 0}=(\mathrm{a})-0.4$ and (b) $0.0 \mathrm{MHz}$ while other parameters are the same as in Fig. 11. The insets show the relative difference between the reflection spectra obtained with the two approaches.

It is worth noting here that the reflection and transmission properties so far examined rely on atomic samples with a uniform density. In most cases the gaseous samples of ultracold atoms are nonuniform and possibly smooth. The Bloch wave-vector dispersion and the photonic bandgap structure depend not only on the standingwave (SW) pump field but also on the local atomic density. For a nonuniform sample, if it is simply composed of several uniform slabs having different atomic densities, these slabs will possess different bandgaps so that one cannot draw a single plot of the bandgap structure. Consequently, the probe spectra of reflection and transmission will change when a uniform sample is replaced by a nonuniform one. In practice, how significant will be the modification of the results for reflection and transmission depends a lot on the profile of the atomic density along the propagation direction. In case the sample has rather thin entrance and exit boundary layers (where the density changes rapidly) and a thick interior layer (where the density changes only a little and very slowly), we expect that the average density of the interior layer determines the bandgap structure and the boundary layers modify only slightly the spectra by suppressing the rapid oscillations at the edges of the stop band. More generally, if two or more thick layers with a nearly constant density can be identified, within each of them a distinct band structure can develop and the spectra would depend on how the respective stop bands are aligned. If the probe pulse is entirely contained in at least one of these stop bands (and the corresponding layer is thick enough) it will be reflected entirely although maybe partly distorted. In the complete general case of arbitrary variations of density, we still expect to have high reflectivity in a certain frequency region of quasi-stop-band, and to flexibly control the flow of the probe pulse by modifying the SW coupling field. 


\section{SUMMARY}

We here address the issue of the optical response of an ensemble of ultracold ${ }^{87} \mathrm{Rb}$ atoms driven by a static laser field in a SW configuration. This has been successfully modeled through a dressed atomic susceptibility based two-mode approximation whose validity has been verified by direct comparison with the Maxwell-Liouville equations in the limit of a time-independent coupling field. A fairly straightforward Fourier decomposition of the susceptibility enables us to obtain analytical expressions for the optical Bloch wave-vector dispersion as well as for the reflectivity and transmissivity spectra of the atomic ensemble. This in turn provides a sound explanation for nearly perfect reflection exhibited by $\mathrm{cw}$ probe fields of frequencies falling within the optically induced photonic bandgap. We further investigate the optical response of the atomic sample both to a long square and to a Gaussian pulse. We find that pulse propagation through the cold atomic ensemble can be fully and all-optically controlled so that for Gaussian pulses falling within the the bandgap, for instance, reflection occurs with little loss and deformation, yet with a time delay and appreciable penetration into the medium. Outside the bandgap region both reflected and transmitted Gaussian pulses suffer instead significant loss and deformation. We expect that upon a suitable extension to solid materials, such as impurity doped crystals and semiconductor compounds, the alloptical control mechanism examined here may be valuable in improving light storage efficiencies in solid quantum memory devices or more generally to further quantum information solid state solutions.

\section{ACKNOWLEDGMENTS}

This work is supported by the Program for New Century Excellent Talents in University (NCET-06-0309), the Program for Distinguished Young Scholars in Jilin Province (20070121), the 973 program (2006 CB 921103), and the PRIN 2006-021037 grant of Ministero della Istruzione, della Universita e della Ricerca (MIUR) Italy. J.-H. Wu is grateful for the hospitality at Scuola Normale Superiore in Pisa.

\section{REFERENCES AND NOTES}

1. S. E. Harris, "Electromagnetically induced transparency," Phys. Today 50, 36-42 (1997).

2. M. Fleischhauer, A. Imamoglu, and J. P. Marangos, "Electromagnetically induced transparency: optics in coherent media," Rev. Mod. Phys. 77, 633-673 (2005).

3. L. V. Hau, S. E. Harris, Z. Dutton, and C. H. Behroozi, "Light speed reduction to 17 meters per second in an ultracold atomic gas," Nature (London) 397, 594-598 (1999).

4. A. V. Turukhin, V. S. Sudarshanam, M. S. Shahriar, J. A. Musser, B. S. Ham, and P. R. Hemmer, "Observation of ultraslow and stored light pulses in a solid," Phys. Rev. Lett. 88, 023602 (2001).

5. M. Fleischhauer and M. D. Lukin, "Dark-state polaritons in electromagnetically induced transparency," Phys. Rev. Lett. 84, 5094-5097 (2000).

6. C. Liu, Z. Dutton, C. H. Behroozi, and L. V. Hau, "Observation of coherent optical information storage in an atomic medium using halted light pulses," Nature (London) 409, 490-493 (2001).
7. J. J. Longdell, E. Fraval, M. J. Sellars, and N. B. Manson, "Stopped light with storage times greater than one second using electromagnetically induced transparency in a solid," Phys. Rev. Lett. 95, 063601 (2005).

8. S. E. Harris and L. V. Hau, "Nonlinear optics at low light levels," Phys. Rev. Lett. 82, 4611-4614 (1999).

9. D. A. Braje, V. Balic, G. Y. Yin, and S. E. Harris, "Low-lightlevel nonlinear optics with slow light," Phys. Rev. A 68, 041801(R) (2003).

10. A. B. Matsko, A. Novikova, G. R. Welch, and M. S. Zubairy, "Enhancement of Kerr nonlinearity by multiphoton coherence," Opt. Lett. 28, 96-98 (2003).

11. C. Ottaviani, D. Vitali, M. Artoni, F. Cataliotti, and P. Tombesi, "Polarization qubit phase gate in driven atomic media," Phys. Rev. Lett. 90, 197902 (2003).

12. S. Rebic, D. Vitali, C. Ottaviani, P. Tombesi, M. Artoni, F. Cataliotti, and R. Corbalan, "Polarization phase gate with a tripod atomic system," Phys. Rev. A 70, 032317 (2004).

13. S. E. Harris and Y. Yamamoto, "Photon switching by quantum interference," Phys. Rev. Lett. 81, 3611-3614 (1998).

14. A. Andre and M. D. Lukin, "Manipulating light pulses via dynamically controlled photonic band gap," Phys. Rev. Lett. 89, 143602 (2002).

15. M. Bajcsy, A. S. Zibrov, and M. D. Lukin, "Stationary pulses of light in an atomic medium," Nature (London) $\mathbf{4 2 6}$ 638-641 (2003).

16. A. Andre, M. Bajcsy, A. S. Zibrov, and M. D. Lukin, "Nonlinear optics with stationary pulses of light," Phys. Rev. Lett. 94, 063902 (2005).

17. B. S. Ham, "Spatiotemporal quantum manipulation of traveling light: quantum transport," Appl. Phys. Lett. 88, 121117 (2006).

18. F. E. Zimmer, A. Andre, M. D. Lukin, and M. Fleischhauer, "Coherent control of stationary light pulses," Opt. Commun. 264, 441-453 (2006).

19. S. A. Moiseev and B. S. Ham, "Generation of entangled lights with temporally reversed photon wave packets," Phys. Rev. A 71, 053802 (2005).

20. K. R. Hansen and K. Molmer, "Trapping of light pulses in ensembles of stationary lambda atoms," Phys. Rev. A $\mathbf{7 5}$, 053802 (2007).

21. K. R. Hansen and K. Molmer, "Stationary light pulses in ultracold atomic gases," Phys. Rev. A 75, 065804 (2007).

22. K. Sakoda, Optical Properties of Photonic Crystals (Springer, 2001).

23. X.-M. Su and B. S. Ham, "Dynamic control of the photonic band gap using quantum coherence," Phys. Rev. A 71, 013821 (2005).

24. D. Petrosyan, "Tunable photonic band gaps with coherently driven atoms in optical lattices," Phys. Rev. A 76, 053823 (2007).

25. M. Artoni and G. C. La Rocca, "Optically tunable photonic stop bands in homogeneous absorbing media," Phys. Rev. Lett. 96, 073905 (2006)

26. Q.-Y. He, Y. Xue, M. Artoni, G. C. La Rocca, J.-H. Xu, and J.-Y. Gao, "Coherently induced stop-bands in resonantly absorbing and inhomogeneously broadened doped crystals," Phys. Rev. B 73, 195124 (2006).

27. Q.-Y. He, J.-H. Wu, T.-J. Wang, and J.-Y. Gao, "Dynamic control of the photonic stop bands formed by a standing wave in inhomogeneous broadening solids," Phys. Rev. A 73, 053813 (2006).

28. J. P. Prineas, J. Y. Zhou, J. Kuhl, H. M. Gibbs, G. Khitrova, S. W. Koch, and A. Knorr, "Ultrafast ac Stark effect switching of the active photonic band gap from Braggperiodic semiconductor quantum wells," Appl. Phys. Lett. 81, 4332-4334 (2002)

29. S. M. Sadeghi, W. Li, and H. M. van Driel, "Coherently induced one-dimensional photonic band gap," Phys. Rev. B 69, 073304 (2004).

30. F. Silva, J. Mompart, V. Ahufinger, and R. Corbalan, "Electromagnetically induced transparency in Dopplerbroadened three-level systems with resonant standingwave drive," Europhys. Lett. 51, 286-292 (2000). 
31. For the atomic samples considered here $\lambda_{c}$ coincides, however, with the vacuum pump wave vector as the dielectric constant experienced by the pump is essentially unity.

32. K. Riley, M. Hobson, and S. Bence, Mathematical Methods for Physics and Engineering, 3rd ed. (Cambridge U. Press, 2006).

33. N. Ashcroft and D. Mermin, Solid State Physics (Saunders College, 1976).

34. M. Artoni, G. La Rocca, and F. Bassani, "Resonantly absorbing one-dimensional photonic crystals," Phys. Rev. E 72, 046604 (2005).

35. By replacing $k_{p} \rightarrow\left(\omega_{p} / c\right)$ and $\kappa \rightarrow k_{c}$ in Eq. (11) in the simplified case where $\chi_{0}$ and $\chi_{1}$ are real and frequency independent, one obtains $\omega_{p}^{2} \rightarrow\left(k_{c} c\right)^{2} /\left(1+\chi_{0} \mp \chi_{1}\right)$ yielding the upper and lower edge of the frequency stop band at the Brillouin zone boundary $\pi / a$. The width of such a photonic bandgap is directly proportional to $\chi_{1}$.

36. M. Born and E. Wolf, Principles of Optics, 7th (expanded) ed. (Cambridge U. Press, 1999).

37. The optical coherence in Eq. (22) contributes with the two components $\rho_{31}^{\{-1,0\}}$ while the probe polarization $P_{p}$ has an expression analogous to the one for $E_{p}$ in Eq. (21) where the "forward" polarization component $P_{p+}=\epsilon_{0}\left(\chi_{0} E_{p+}+\chi_{1} E_{p-}\right)$ and a similar one for the "backward" component $P_{p-}$, which is obtained upon interchanging $\{+\leftrightarrow-\}$ in $P_{p+}$. 